\title{
Determining Whether Tai Chi Chuan is Related to the Updating Function in Older Adults: Differences Between Experts and Novices
}

\section{Yuan Yang}

Beijing Normal University

Tingting Chen

Beijing Dance Academy

\section{Xiaoxia Yuan}

Capital University of Physical Education and Sports

Xiaoke Zhong

Capital University of Physical Education and Sports

Shoufu Yan

Capital University of Physical Education and Sports

Changhao Jiang ( $\nabla$ jiangchanghao@cupes.cn )

Capital University of Physical Education and Sports

\section{Research Article}

Keywords: Updating function, Tai Chi Chuan, older adults

Posted Date: August 27th, 2021

DOI: https://doi.org/10.21203/rs.3.rs-783067/v1

License: (c) (1) This work is licensed under a Creative Commons Attribution 4.0 International License. Read Full License 


\section{Abstract}

Background: Tai Chi Chuan (TCC) is one effective method used to delay cognitive decline in older adults. However, the relationship between the long-term practice of TCC and the crucial working memory updating function of the brain in the elderly has not been comprehensively researched.

Objective: This cross-sectional study explores how behavioral performance in a laboratory setting is related to long term practice of TCC on the updating function in older people using reaction times (RTs) of $\mathrm{N}$-back tasks.

Methods: 26 healthy elderly people participated in this experiment. According to the length of time TCC was practiced, 13 subjects in the TCC group had more than 5 years' experience of TCC exercise, and 13 elderly subjects who had not been systematically exposed to mind-body exercise were assigned to the novice group. The N-back task was administered to every participant to evaluate the updating function.

Results: The TCC group had faster RTs compared to the novice group $(\mathrm{p}<0.05)$; For the 1 -back task, the TCC group showed faster RTs than the novice group. For the 2-back task, the novice group exhibited faster RTs than the TCC group.

Conclusions: TCC experts exhibit greater updating function as compared to TCC novices. Long-term TCC appears to be positive associated with the updating function of older adults, making it an effective exercise to maintain cognitive function in the elderly.

\section{Introduction}

Authors Problems facing the aging population have become very prominent. Elderly persons are vulnerable to decline in physical function and a high incidence of disease [1]. In addition to the decline of physical ability, the decline of cognitive function seriously impairs quality of life for the elderly. Previous studies have shown that cognitive decline was as-sociated with age-induced neurodegeneration and reduced brain activation compared to younger adults [2-3].

Executive function decline is one of the manifestations of cognitive decline. Executive function refers to the coordination of various resources and the processing required by the control system to accomplish specific goals when the brain completes complex cognitive tasks [4]. The executive function includes working memory (WM) and other sub-components [5]. Updating is part of WM, and plays an important role in the whole process of advanced cognition [6]. With age, the updating function of the elderly gradually declines. $\mathrm{N}$-back is a behavioral experimental paradigm for detecting the updating function, widely used in neurocognitive research [7]. Age-related changes in brain characteristics can be effectively demonstrated by performing $\mathrm{N}$-back cognitive tasks [8].

Research has shown that the decline of WM was significantly related to cognitive impairment, such as Alzheimer's disease [9-11]. It is expected that there will be 75 million elderly people suffering from 
dementia globally by 2030 [12]. Therefore, the discovery of effective measures to intervene in aging and delay WM decline has become the focus of current research in the cognitive neuroscience field. Both physiological impact and psychological benefits should be focused on when choosing the maintenance strategies [13].

Mindfulness has a positive effect on the executive function of the elderly and may be beneficial for the performance of the WM in older people. The Tai Chi Chuan (TCC) is accompanied by concentration and breathing control in order to improve physical strength, balance, flexibility and promote physical health [14-16]. TCC is gentle, slow, coherent, relaxed and involves stretching. Compared with other training methods that emphasize endurance and strength, TCC is safer and more suitable for the elderly to participate in [17].

Previous studies have shown that TCC can improve the performance of the elderly in N-back tasks. Studies by Mortimer et al. [18], showed that healthy elderly people who completed 40 weeks of Tai Chi exercise performed better in working memory tasks and demonstrated higher activation levels of brain areas related to cognitive function. Lam et al. [19], randomly allocated elderly subjects to experimental and control groups in which the experimental group was required to complete Tai Chi exercise three times a week, for 30 minutes each time, for one year, while the control group only performed stretching exercises. After 5 months of exercise, the test scores of the experimental group's working memory task improved, and continued to improve, after one year.

Although many studies have verified the effect of TCC on improving WM, the results have not been consistent. For example, a 20-week TCC intervention study conducted by Hall et al. [20], showed that there was no significant change in the behavioral performance of the elderly in the cognitive task after TCC intervention. However, studies have found that mindfulness meditation exercise has a specific effect on executive function, the most specific inhibitory component, but the benefits to updating and shifting domains are variable [21-22]. It is not clear whether the benefit of mindfulness in improving executive function is a selective improvement of one sub-ability or an improvement of the whole. At present, the effect of mindfulness on the results is inconsistent, and needs to be further verified. Zeidan et al. [23] indicated that the brief mindfulness training improves $\mathrm{N}$-back task performance and has favorable effects on the number of correct responses over time compared to control group. Thus, based on the specific effects of brief mindfulness, this type of intervention could improve the updating function, and elevated sustained accuracy as time pass by. Based on this result, it is necessary to investigate the relationship between long-term mindfulness training and executive function specific subability.

In order to address these knowledge gaps, we conducted this study to explore the possible characteristics of TCC, an oriental mindfulness exercise, in improving the up-dating function of aging elderly people. A cross-sectional study design and a behavioral experiment ( $\mathrm{N}$-back) were used to examine the relationship between TCC with the updating function of the elderly.

Based on the above theories and findings, this study proposes the following hypotheses: 


\section{Hypothesis 1}

(H1). TCC experts exhibit greater updating function as compared to age-matched and gender-matched, non-sedentary, TCC novices.

\section{Hypothesis 2}

$(\mathrm{H} 2)$. There is a positively correlation between Long-term TCC and up-dating function. With the increasing of TCC training period, the working memory updating function is also enhanced.

\section{Materials And Methods \\ 2.1. Participants}

Twenty-six (26) participants were recruited from the local community from China. The screening conditions were as follows: (1) no brain trauma, psychiatric history, chronic diseases; (2) educational background of high school and above; (3) right-handed; (4) with normal vision or corrected vision; (5) a Minimum Mental State Examination (MMSE) score $\geq 24$; (6) spent 1 hour a day in exercise. All the subjects were required to not drink alcohol, stay up late or take part in heavy exercise one day be-fore the experiment, so as to ensure adequate sleep and a healthy cognitive state.

Two groups of subjects were selected according to TCC experience. Thirteen (13) sub-jects in the TCC group had more than 5 years of TCC exercise experience ( 60 min per session for 3 days per week; average age of $65.23 \pm 1.48$ years; TCC for $5.77 \pm 0.73$ years); Thirteen (13) elderly subjects who had not been systematically exposed to mind-body exercise were assigned to the control group (average age of 65.15 \pm 3.69 ).

The Capital University of Physical Education and Sports (CUPES) Ethics Committee (approval No. CUPES2018-06-15-01) approved the study, and all participants signed an informed consent form before participating in the experiment. The whole experimental process is shown in Fig. 1.

\subsection{Procedures}

This experiment ran the N-back task paradigm using E-prime 2.0 on a 14-inch notebook computer with $1024 * 768$ resolution, and compiled the $\mathrm{N}$-back experiment task performance with E-prime software. An Nback task paradigm was used to test the updating function. Before the formal experiment began, the subjects were instructed to operate the practice mode according to the required proficiency task test procedures. The formal test begins at the end of the exercise mode. The whole N-back formal test consisted of four blocks, including two 1-back tasks and two 2-back tasks. Each block consisted of a total of 30 tests, and stimulus materials were randomly presented in the center of the computer screen. The stimulus materials for this experiment were English letters (including upper and lower case). In each experiment, a black screen was used for key feed-back. Reaction time was measured during the black 
screen, which was presented for up to $1000 \mathrm{~ms}$ before the next stimulus cycle began. In the 1-back task, participants were re-quired to compare the last letter with the previous one in succession. If the latter letter was the same as the previous letter, they were instructed to press the "F" key, and if different, they were instructed to press the "J" key. In the 2-back task, participants were required to compare the second letter in succession with the second letter in front. If these letters were the same, they were instructed to press the "F" key, and if different, they were instructed to press the "J" key. The entire formal test time was about 5 minutes, and subjects were given 30 seconds rest time between each block, 150 seconds resting time was granted overall. The entire test was carried out in a laboratory environment with quiet surroundings. The main outcome was the reaction times (RTs) in performing N-back tasks. Figure 2 illustrates the testing process of the N-back task.

\subsection{Statistical Analysis}

The experiment was designed as a mixed-model experiment of 2 group types (TCC group, novice group) $\times$ 2 task types (1-back, 2-back), in which group type was the inter-group variable, task type was the intragroup variable, and the RTs were dependent variables. The IBM SPSS 23.0 software was used for statistical analysis. The RTs of the behavioral data were analyzed by repeated measurements and an ANOVA of 2 group types (TCC group, novice group) $\times 2$ task types (1-back, 2-back). If there was a significant interaction, simple effect analysis was used for further statistical analysis. The $p$ value was corrected by the Greenhouse-Geisser method, and the significance level a was set at a value of 0.05 .

\section{Results}

\subsection{Demographic Data Results}

Before the experiment, the demographic variables of the two groups were analyzed (Table 1). They showed no significant differences in age, years of education, MMSE scores and body mass index (BMI) between the three groups $(p>0.05)$, indicating homogeneous demographic characteristics between the groups.

Table 1

Demographic data of the participants $(M \pm S D)$.

\begin{tabular}{|llll|}
\hline Factor & TCC Group & Novice Group & $\mathbf{p}$ \\
\hline Age (years) & $65.23 \pm 1.48$ & $65.15 \pm 3.69$ & 0.95 \\
\hline BMI & $24.45 \pm 1.34$ & $24.16 \pm 1.26$ & 0.58 \\
\hline Education (years) & $12.92 \pm 1.76$ & $13.54 \pm 2.03$ & 0.42 \\
\hline MMSE Score & $27.08 \pm 0.86$ & $26.77 \pm 1.67$ & 0.45 \\
\hline TCC = Tai Chi Chuan group. & & \\
\hline
\end{tabular}

\subsection{RTs Analysis of N-back Task}


The sample was tested for normal distribution. The RTs of the N-back task are shown in Table 2. When analyzing whether TCC exercise affects the updating function of individuals, the RTs of the N-back task were tested as a dependent variable. The results showed that the main effect of task type (1-back and 2back task) was significant $[F(1,24)=23.487, p=0.000]$, and the main effect of the groups (TCC group, Novice group) was significant, indicating there were differences in RTs between the two groups $[F(1,24)=$ $11.000, p=0.003]$, and the interaction between group and task type was significant $[F(1,24)=4.686, p=$ $0.041]$.

Table 2

$\mathrm{N}$-back task RTs of participants (M $\pm \mathrm{SD})$.

\begin{tabular}{|lllc|}
\hline Task & TCC & Novice & n \\
\hline 1-back & $396.06 \pm 49.22^{*}$ & $429.18 \pm 27.92$ & 13 \\
\hline 2-back & $426.61 \pm 58.84^{*}$ & $509.06 \pm 67.84$ & 13 \\
\hline \multicolumn{4}{|l}{ TCC = Tai Chi Chuan; RTs = Reaction times; ${ }^{*}, \mathrm{p}<0.05$} \\
\hline
\end{tabular}

Further, the simple-effects analysis showed that under 1-back task conditions, the RTs in the TCC group were faster than those in the novice group, and the difference was significant $(p<0.05)$; the 2-back task RTs in the TCC group were faster than those in the novice group, and the difference was significant $(p<0.05)$ (Figure 3). Therefore, the long-term TCC appears to be associated with the updating function of the elderly participants.

\section{Discussion}

This study aimed to examine the long-term effects of TCC exercise on the updating aspect of the WM performance in older adults in behavioral domains between experts and novices.

The results showed that compared with the novice group, the TCC group had faster RTs in completing the $\mathrm{N}$-back task, suggesting that TCC could promote the updating function in the elderly. This result is consistent with the existing study. Man et al. [24], recruited 135 elderly community subjects over 60 years of age to conduct a cross-sectional study. According to exercise habits, the subjects were assigned to the Tai Chi group, the general exercise group or the control group, and the WM of the subjects was examined by using relevant behavioral tasks. The results showed that the subjects who habitually performed Tai Chi exercise had better WM.

The present study showed that long-term practice of TCC could improve the working memory updating function of the elderly. We also considered the psychological mechanism of the cognitive benefits of this exercise, taking into account the characteristics of the N-back task and TCC. Combined with the behavioral data in this study, it was found that compared with the 2-back task, the RTs of the subjects in the 1-back task was significantly lower, which indicated that subjects needed to mobilize more cognitive resources to process more complex information. These cognitive performances can be improved through exercise and cognitive training. Therefore, long-term TCC may improve the updating function. 
TCC is an oriental mindfulness exercise that integrates flexibility and coordination, and its exercise mechanisms are closely related to the WM of the elderly [25]. It requires the participant to complete cognitive activities, such as visual space processing, motion recall and task switching, while exercising. Thus, TCC is both physical and mental exercise, containing both aerobic exercise and cognitive training [26]. Studies have shown that both aerobic exercise and cognitive training can improve cognitive performance [27]. In addition, the complex technical movements and organizational forms of TCC are closely related to the updating function of the elderly. This study also showed that compared with novices, subjects who had long-term practice of TCC had faster RTs in performing complex updating tasks. In the practice of TCC, the spatial orientation changes greatly. When an elderly person completes the action of different spatial orientation, they should quickly update the spatial orientation for the next action, so as to make the correct maneuver, which promotes the development of the updating function. In order to improve the updating function, it is helpful for elderly people to arrange the whole set of TCC routines on the basis of established movements. They need to combine the whole set of TCC routines based on each one they have learned. When the former movement is completed, it should be updated quickly. This study shows that long-term practice of TCC could improve the performance of the elderly in the $\mathrm{N}$-back tasks, and may be an effective way to maintain the updating function.

TCC involves mindful breathing regulation where individuals focus on breathing in and out. In the process of breathing, the mind is directed to intentionally scan the body in order to influence psychological and physiological states [28]. The RTs of N-back task in this study showed that long-term TCC appears to be associated with better working memory updating function. The TCC experts are all the people who have received systematic professional TCC teaching, and have more than 5 years of training experience. They can really integrate breathing regulation into daily practice and may obtain the psychological and physiological benefits. The electrical activity in the human piriform cortex and limbic-related brain areas are synchronized by natural breathing [29]. The amygdala and hippocampus are closely related to working memory updating function, so respiratory regulation may improve working memory.

TCC contains a meditation component, and studies have shown that meditation im-proves attention. When Tang et al. [30] recruited 40 subjects for a 5-day meditation intervention experiment, the subjects showed improvements in attention, decreased anxiety, depression, anger, and fatigue, and an increase in immunoreactivity. In addition, TCC is a mindfulness exercise practiced in the natural environment. Research shows that mind-fulness exercise in the natural environment has a stronger effect on attention concentration [31]. The improvement of attention also helps the subjects to concentrate their cognitive resources to complete the $n$-back task. This study found that long-term TCC participants were associated with better N-back task performance. TCC requires a relaxed body, calm mind and mind-body unity, and also advocates guiding movement with mind [32].

\subsection{Limitations of the Study}

We would like to acknowledge some limitations of this study. Firstly, this study was a cross-sectional study and could not explain the causal relationship. Secondly, due to the limitation of experimental 
conditions, this study only collected behavioral data and no brain imaging techniques, such as ERP, fNIRS or fMRI, were utilized. It is hoped that future research can employ these brain im-aging techniques to comprehensively evaluate the effect of long-term mind-body exercise on performance. In addition, mindfulness also has an impact on emotion and stress. Future research can consider adding these factors to comprehensively consider the overall positive impact of mindfulness on the elderly. Appling these methods may provide a more theoretical basis for maintaining the cognitive function of the elderly so that they may be provided with rational and efficient exercise prescriptions.

\section{Conclusions}

Compared with the novice group, long-term practice of TCC in the experimental group was shown to potentially improve the updating function of the elderly. There is a positive correlation between the TCC and updating function, which indicates the potential positive effect of TCC exercise. TCC is an oriental mindfulness exercise, which may be an effective way to maintain cognitive function of the elderly.

\section{List Of Abbreviations}

Tai Chi Chuan (TCC); Reaction Times (RTs); Working Memory (WM); Minimum Mental State Examination (MMSE); Body Mass Index (BMI)

\section{Declarations}

Ethics approval and consent to participate: The Capital University of Physical Education and Sports (CUPES) Ethics Committee (approval No. CUPES-2018-06-15-01) approved this study, and all participants signed an informed consent form before participating in the experiment. All methods were carried out in accordance with relevant guidelines and regulations

Consent for publication: Not applicable.

Availability of data and materials: The datasets used and/or analysed during the current study are available from the corresponding author on reasonable request.

Competing interests: The authors declare that they have no competing interests.

Funding:This work was supported by Beijing Natural Science Foundation of China (No. 5212002), the Open Research Fund of the National Center for Protein Sciences at Peking University in Beijing (No. KF202102), and the National Natural Science Foundation (NNSF) of China under Grant No. 31771244.

Authors' Contributions: Conceptualization, Y.Y., S.Y. and C.J.; software, X.Y.; formal analysis, Y.Y., S.Y. and CJ; resources, C.J.; data curation, Y.Y., T.C. X.Z. and X.Y.; writing-original draft preparation, Y.Y.; writingreview and editing, Y.Y., S.Y., X.Y., X.Z., T.C. and C.J.; project administration, C.J.; funding acquisition, C.J. All authors read and approved the final manuscript. 
Acknowledgments: This work was open manuscript to be reviewed Research Fund of the State Key Laboratory of Cognitive Neuroscience and Learning to Changhao Jiang. The experiments comply with the current laws of the country in which they were performed.

\section{References}

1. Monica, M., Ballesteros, S.(2018). Does physical exercise improve perceptual skills and visuospatial attention in older adults? A review. European Review of Aging and Physical Activity, 2018, 15, 2.

2. Herrmann, M.J., Langer, J.B.M., Jacob, C., Ehlis, A.C., Fallgatter, A.J. (2008). Reduced prefrontal oxygenation in Alzheimer disease during verbal fluency tasks. American Journal of Geriatric Psychiatry, $16,125-135$.

3. Richter, M.M., Herrmann, M.J., Ehlis, A.C., Plichta, M.M., Fallgatter, A.J. (2007). Brain activation in elderly people with and without dementia: Influences of gender and medication. World Journal of Biological Psychiatry,2007,8,7.

4. Perner, J., Lang, B. (1999). Development of theory of mind and executive control. Trends in Cognitive Sciences,3,337-344.

5. Smith, E.E. (1999). Storage and executive processes in the frontal lobes. Science,283,1657-1661.

6. Morey, C.C., Cowan, N., Morey, R.D., Rouder, J.N. (2011). Flexible attention allocation to visual and auditory working memory tasks: manipulating reward induces a trade-off. Attention Perception \& Psychophysics,73,458-472.

7. Perrig, W.J. (2010). The concurrent validity of the N-back task as a working memory measure. Memory, 18,394-412.

8. Redick, T.S., Lindsey, D.R.B. (2013). Complex span and n-back measures of working memory: a metaanalysis. Psychonomic Bulletin and Review,20,1102-1113.

9. Goodman, M.S., Kumar, S., Zomorrodi, R., Ghazala, Z., Cheam, A.S.M., et al. (2018). Theta-Gamma coupling and working memory in Alzheimer's dementia and Mild cognitive impairment. Frontiers in Aging Neuroscience,10,1-10.

10. Stopford, C.L., Thompson, J.C., Neary, D., Richardson, A.M.T., Snowden, J.S. (2012). Working memory, attention, and executive function in Alzheimer's disease and frontotemporal dementia. Cortex,48,429-446.

11. Whitwell, J.L., Jones, D.T., Duffy, J.R., Strand, E.A., Machulda, M.M., et al. (2015). Working memory and language network dysfunctions in logopenic aphasia: a task-free fMRI comparison with Alzheimer's dementia. Neurobiology Aging,36,1245-1252. 
12. Steen, J.C., Gregers, H.S., Gunhild, W., Hviid, S.A. (2015). Biochemical Markers of Physical Exercise on Mild Cognitive Impairment and Dementia: Systematic Review and Perspectives. Frontiers in Neurology,6,187.

13. Yang, Y., Chen, T., Shao, M., Yan, S., Yue, G.H., et al. (2020). Effects of Tai Chi Chuan on Inhibitory Control in Elderly Women: An fNIRS Study. Frontiers in Human Neuroscience,13,476.

14. Liu, J., Xie, H.H., Liu, M., Wang, Z.B., Zou, L.Y., et al. (2018). The effects of TaiChi on heart rate variability in older Chinese individuals with depression. International Journal of Environment Research and Public Health, 15,2771.

15. Zhou, S.W., Zhang, Y.J., Kong, Z.W., Loprinzi, P.D., Hu, Y., et al. (2019). The effects of Tai Chi on markers of atherosclerosis, lower-limb physical function, and cognitive ability in adults aged over 60: A randomized controlled trial. International Journal of Environment Research and Public Health,16,753.

16. Zou, L.Y., Zhang, Y.J., Sasaki, J,E., Yeung, A.S., Yang, L., et al. (2019). Wuqinxi Qigong as an alternative exercise for improving risk factors associated with metabolic syndrome: A Meta-Analysis of ran-domized controlled trials. International Journal of Environment Research and Public Health,16,1396.

17. Zheng, G.H., Liu, F.W., Li, S.Z., Huang, M.M., Tao, J., et al. (2015). Tai Chi and the protection of cognitive ability: a systematic review of prospective studies in healthy adults. American Journal of Preventive Medicine, 49,89-97.

18. Mortimer, J.A., Ding, D., Borenstein, A.R., Charles, D.C., Guo, Q.H., et al. (2012). Changes in brain volume and cognition in a randomized trial of exercise and social interaction in a community-based sample of non-demented Chinese elders. Journal of Alzheimers Disease,30,757-766.

19. Lam, L.C.W., Chau, R.C.M., Wong, B.M.L., Fung, A.W.T., Lui, V.W.C., et al. (2011). Interim follow-up of a randomized controlled trial comparing Chinese style mind body (TaiChi) and stretching exercises on cognitive function in subjects at risk of progressive cognitive decline. International Journal of Geriatric Psychiatry,26,733-740.

20. Hall, C.D., Miszko, T., Wolf, S.L. (2009). Effects of Tai Chi intervention on dual-task ability in older adults: a pilot study. Archives of Physical Medicine and Rehabilitation,90,525-529.

21. Gallant, S.N. (2016). Mindfulness meditation practice and executive functioning: Breaking down the benefit. Conousness and Cognition,40,116-130.

22. Zhou, H.G., Liu, H.V., Deng, Y.L. (2020). Effects of short-term mindfulness-based training on executive function: Divergent but promising. Clinical Psychology \& Psychotherapy,27,672-685.

23. Zeidan, F., Johnson, S.K., Diamond, B.J., Zhann, D., Goolkasian, P. (2010). Mindfulness meditation improves cognition: Evidence of brief mental training. Conousness and Cognition,19,597-605. 
24. Man, D.W.K., Tsang, W.W., Hui-Chan, C.W. (2010). Do older Tai Chi practitioners have better attention and memory function? Journal of Alternative and Complementary Medicine,16,1259-1260.

25. Chang, Y.K., Nien, Y.H., Tsai, C.L., Etnier, J.L. (2010). Physical activity and cognition in older adults: The potential of Tai Chi Chuan. Journal of Aging and Physical Activity,18,451-472.

26. Chen, T.T., Yue, G.H., Tian, Y.X., Jiang, C.H. (2017). Baduanjin mind-body intervention improves the executive control function. Frontiers in Psychology,7,2015.

27. Tao, J., Liu, J., Liu, W., Huang, J., Xue, X., et al. (2017). Tai Chi Chuan and Baduanjin increase grey matter volume in older adults: A brain imaging study. Journal of Alzheimers Disease,60,389.

28. Utay, J., Miller, M. (2006). Guided imagery as an effective therapeutic technique: a brief review of its history and efficacy research. Journal of Applied Psychology, 33, 40-43.

29. Zelano, C., Jiang, H., Zhou, G., Arora, N., Schuele, S., et al. (2017). Nasal respiration entrains human limbic oscillations and modulates cognitive function. Journal of Neuroscience. 2017,36,12448-12467.

30. Tang, Y.Y., Ma, Y., Wang, J., Fan, Y., Feng, S., et al. (2007). Short-term meditation training improves attention and self-regulation[J]. Proceedings of the National Academy of Sciences of the United States of America, 104,17152-17156.

31. Pfeifer, E., Fiedler, H., Wittmann, M. (2019). Increased relaxation and present orientation after a period of silence in a natural sur-rounding. Nordic Journal of Music Therapy,29,75-92.

32. Cui, H., Wang, Q.Y., Pedersen, M., Wang, Q., Lv S.J., et al. (2019). The safety of tai chi: a meta-analysis of adverse events in randomized controlled trials. Contemporary Clinical Trials,82,85-92.

\section{Figures}




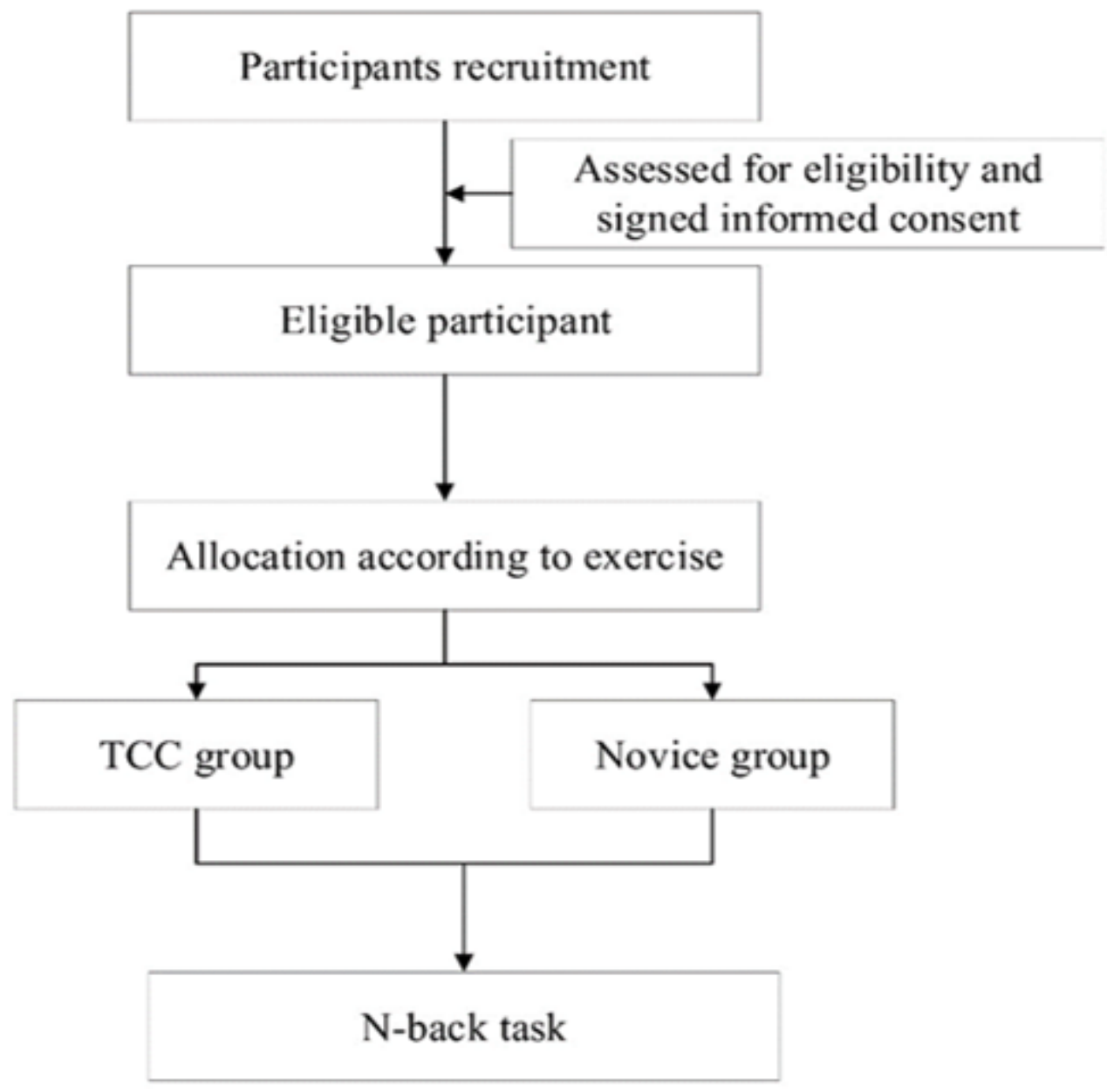

Figure 1

Flow diagram of study design. 


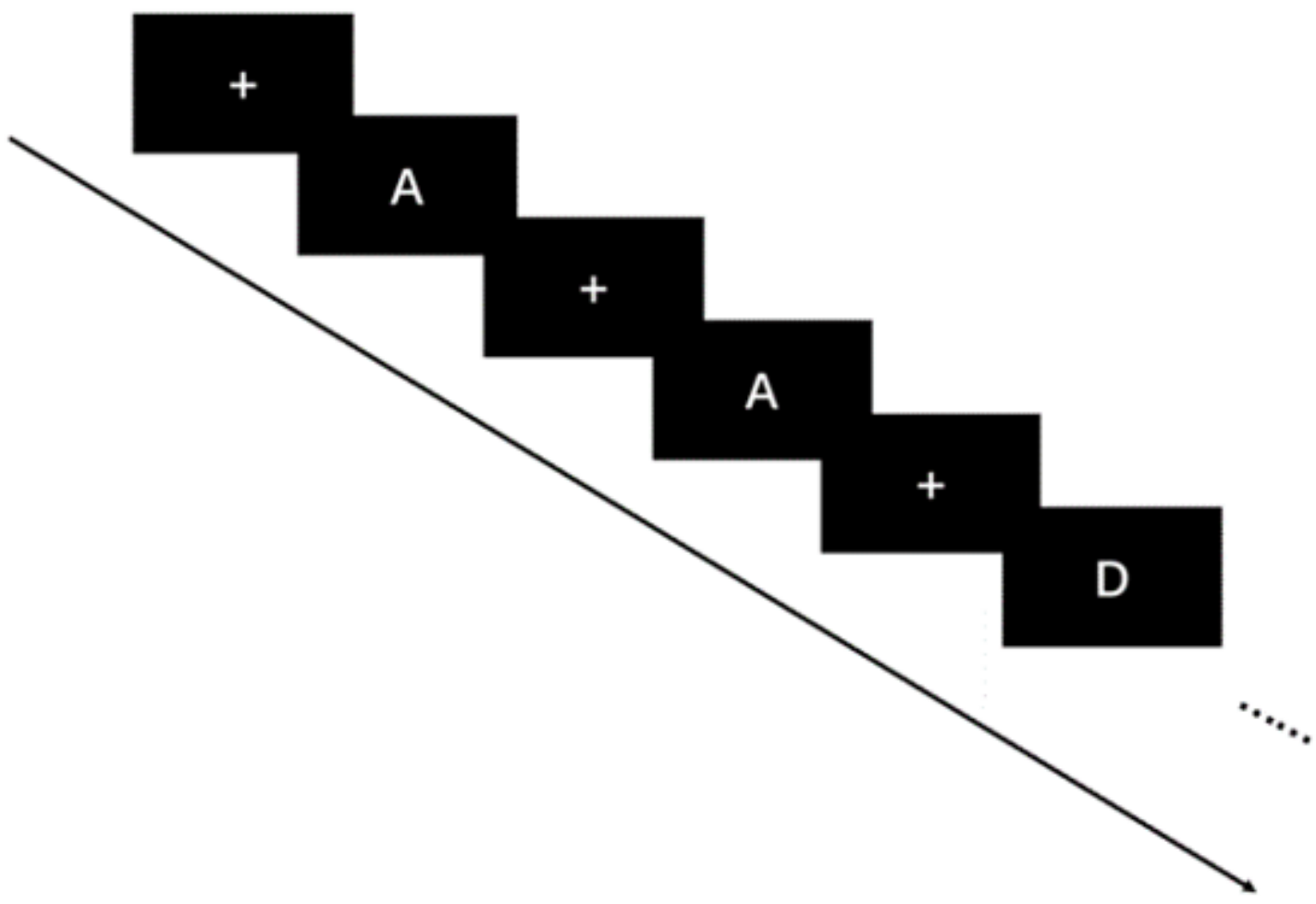

Figure 2

In the 1-back task, participants need to determine whether a letter appears the same as the one before it. In the 2-back task, participants need to determine whether a letter appears the same as the two before it. If the same, press " $F$ "; if different, press " $J$ ". The reaction time is $1000 \mathrm{~ms}$. 


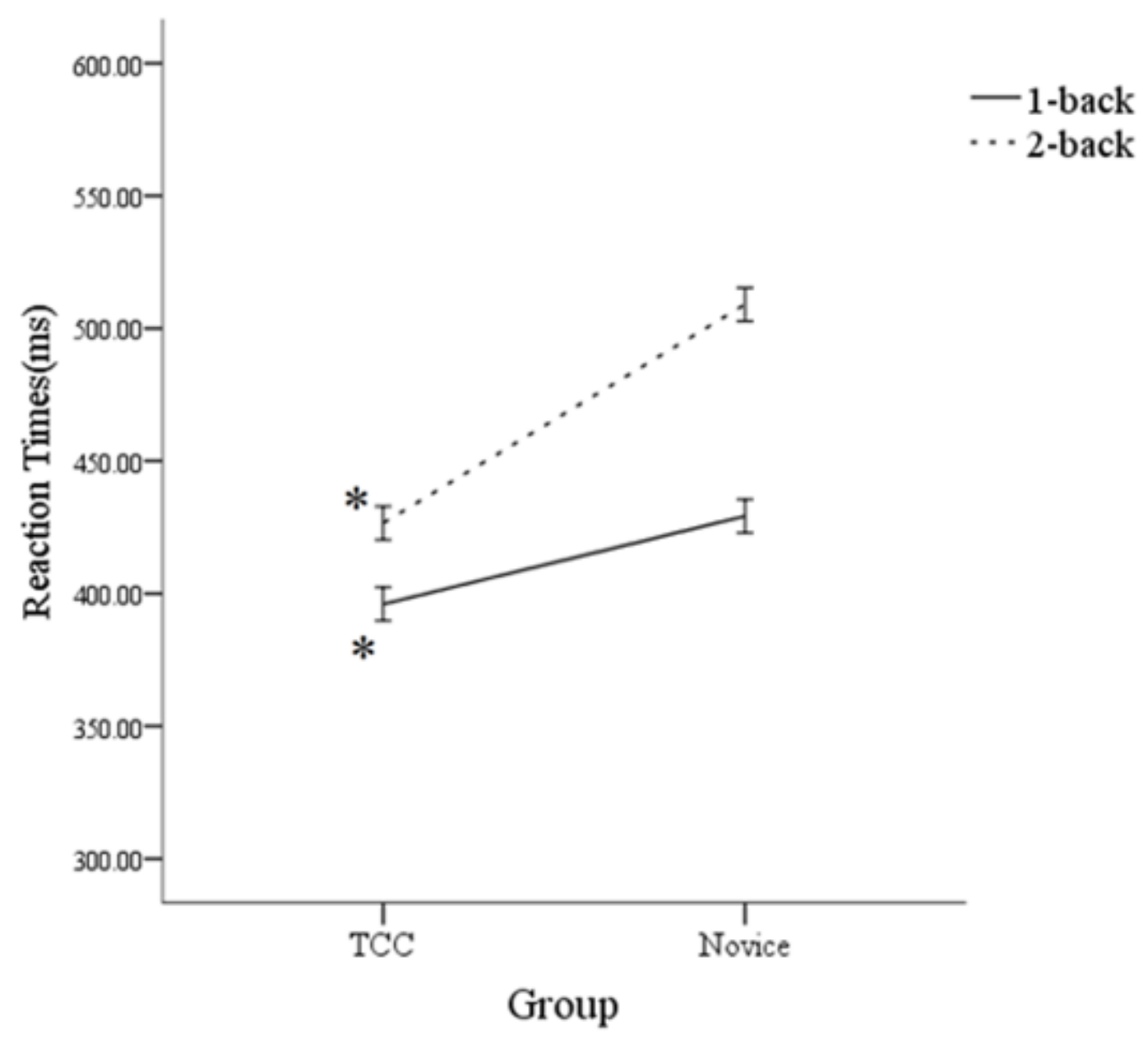

Figure 3

Comparison of RTs between the three groups (TCC vs. Novices) during N-back task. 